\title{
Editorial: \\ Perspectivas Actuales en el Estudio de la Dislexia Evolutiva
}

\section{Manuel Soriano Ferrer}

Departamento de Psicología Evolutiva y de la Educación, Universidad de Almería

\section{España}

msorian@ual.es 


\section{Editorial}

La dislexia evolutiva constituye un trastorno complejo desde el punto de vista biológico, cognitivo y conductual, que tiene un impacto negativo en la vida académica de las personas que lo sufren. Se trata de una dificultad en el aprendizaje, caracterizada principalmente por un fracaso inesperado en el reconocimiento de las palabras, que se manifiesta en la incapacidad para desarrollar una lectura fluida y sin esfuerzo.

A pesar de los avances producidos desde que se describiera la dislexia evolutiva por primera vez en 1896, sigue estando rodeada de acalorados debates y desacuerdos acerca de cuestiones fundamentales como la definición, la prevalencia, las causas, las manifestaciones, y el tratamiento. Esta es la razón que ha motivado a la Revista Electrónica de Investigación Psicoeducativa y Psicopedagógica a dedicar este número a la dislexia evolutiva. Este número presenta dos características principales. En primer lugar, los artículos que se incluyen en este número se centran en un mismo tema. En segundo lugar, todos estos artículos son el reflejo de la disparidad de opiniones y enfoques que conviven en la actualidad.

Frecuentemente se citan tasas de prevalencia de la dislexia evolutiva que oscilan entre el $5 \%$ y el $20 \%$. Ante esta alta variabilidad, Tim Miles analiza los problemas con los que nos encontramos a la hora de determinar la prevalencia de la dislexia.

Por otro lado, una gran cantidad de investigaciones, desarrolladas desde la medicina y desde la psicología fundamentalmente, han intentado detectar marcadores etiológicos de la dislexia, aunque aún no se han encontrado resultados definitivos, haciendo alusión la literatura especializada a factores de distinta naturaleza. En la actualidad conviven cuatro teorías principales de la dislexia evolutiva, con un amplio corpus de evidencia empírica, capaces de ofrecer una explicación plausible de las manifestaciones centrales de los disléxicos. Dichas teorías pueden ser agrupadas en dos líneas de trabajo antagonistas. De una parte, la que considera que la dislexia está causada exclusiva y directamente por un déficit cognitivo específico. En el otro lado, aquellas teorías que consideran que estos déficit cognitivos serían secundarios a un déficit mucho más general y primario (Soriano, 2004 a). En este contexto, se desarrollan tres de los trabajos del monográfico. En primer lugar, Francisca Serrano y Sylvia Defior, revisan brevemente el estado actual de la dislexia en torno a diferentes tópicos. A continuación, Angela Fawcett y Rod Nicholson se centran en una de las teorías de déficit general más nove- 
dosas de la dislexia - la teoría cerebelar-, que ofrece una explicación más comprensiva de las manifestaciones de la dislexia y sus comorbilidades. Finalmente, José Escoriza analiza en profundidad la hipótesis del déficit fonológico y las implicaciones para la evaluación e intervención.

Por otro lado, diversas investigaciones longitudinales han mostrado la dislexia como un trastorno crónico, con fuerte persistencia de las dificultades lectoras a largo plazo y con consecuencias educativas que trascienden el aprendizaje lector. Además, la dislexia se manifiesta de forma más clara con el desarrollo, es decir, de año en año (Snowling, Goulandris y Defly, 1996; LaBuda y Defries, 1989). Por ello, cada vez son más los autores que el mejor tratamiento de las dificultades lectoras que podemos hacer es la detección temprana y el tratamiento de sus dificultades. En esta línea de trabajo se sitúa el trabajo de Sotiria Tzivinikou donde se analizan las ventajas y limitaciones de los instrumentos de detección temprana de niños en situación de riesgo.

A continuación se incluyen dos trabajos empíricos realizados desde la Universidad de La Laguna que se centran en la caracterización de los problemas que experimentan los disléxicos en castellano. Por un lado, Juan E. Jiménez et al. presentan un trabajo dirigido a determinar si los disléxicos presentan déficit en el procesamiento sintáctico y Mercedes Rodrigo et al., se centran en el rol que las unidades léxicas y subléxicas tienen en el procesamiento ortográfico de los disléxicos españoles.

Otra de las cuestiones a las que nos vamos a referir es a la intervención. En las últimas décadas se ha producido un esfuerzo notable en el desarrollo de programas de intervención y en el análisis de su efectividad sin precedentes en el campo de las dificultades de aprendizaje. Obviamente, este incremento de las investigaciones de intervención no es fortuito, sino que va paralelo a los avances en la caracterización de los problemas que experimentan los disléxicos evolutivos. De hecho, los diferentes programas de intervención dirigidos a recuperar /aminorar el/los mecanismo-s defectuoso-s, que suponemos causa-n el problema lector son el reflejo de las diferentes teorías explicativas de la dislexia evolutiva (Soriano, 2004 b). En el contexto de intervención, la instrucción asistida a través de ordenador (CAI) ha sido reivindicada por numerosos autores en los últimos años. Una de las principales ventajas de los ordenadores para los alumnos con dislexia es que ofrecen refuerzo y práctica, junto con instrucción individualizada con feedback inmediato, características que son esenciales en la enseñanza a estos estudiantes. Así, la metodología de instrucción basada en ordenador, por sus 
características en cuanto a organización, estructuración y formato atractivo (interface) constituye un procedimiento útil para motivar e intensificar la práctica con el fin de lograr la automatización de las subdestrezas elementales como el reconocimiento y escritura de palabras. En esta línea de trabajo, Manuela Torres nos presenta un programa de software multicomponente que se está desarrollando para la intervención en dislexia evolutiva.

Para terminar me gustaría señalar que no se ha logrado plenamente el objetivo que nos propusimos: ofrecer una perspectiva amplia de todas las líneas de investigación. Varios inves-

tigadores declinaron amablemente nuestra invitación. Por este motivo, quiero agradecer con gran entusiasmo a los diferentes autores nacionales e internacionales su participación en este número. Sin vosotros este número no hubiera sido posible.

\section{Referencias}

LaBuda, M.C. y DeFries, J. C. (1989). Differential prognosis of reading-disabled children as a function of gender, socio-economic status, IQ and severity: A longitudinal study. Reading and Writing, 1, 25-36.

Snowling, M. J., Goulandris, N. y Defly, N. (1996). A Longitudinal Study of Reading Development in Dyslexic Children. Journal of Educational Psychology, 88, 653-669.

Soriano, M. (2004 a). Implicaciones educativas del déficit cognitivo de la dislexia evolutiva. Revista de Neurología, 38, 1, 47-52.

Soriano, M. (2004 b). Intervención Psicoeducativa en la dislexia evolutiva. Ponencia presentada al I Congreso Vasco de Dificultades en el Aprendizaje de la Lecto-Ecritura. Bilbao, 8 de Octubre. 